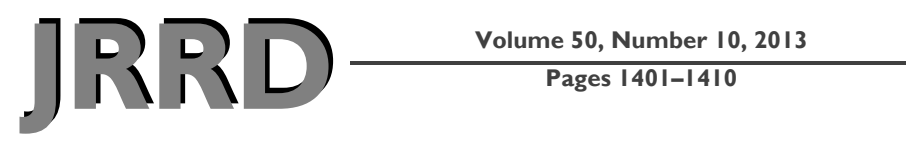

\title{
Objective and subjective measures reflect different aspects of balance in multiple sclerosis
}

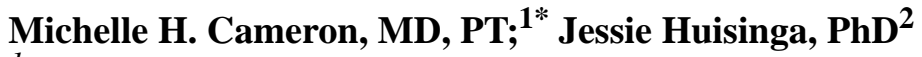 \\ ${ }^{1}$ Department of Neurology, Oregon Health \& Science University, Portland, OR, and Neurology Service, Portland \\ Department of Veterans Affairs Medical Center, Portland, OR; ${ }^{2}$ Landon Center on Aging, University of Kansas Medical \\ Center, Kansas City, KS
}

\begin{abstract}
The objective of this study was to evaluate relationships between subjective and objective measures of balance in multiple sclerosis (MS). In 54 subjects with MS, balance was measured objectively with the Sensory Organization Test (SOT) using dynamic posturography and subjectively with the Activitiesspecific Balance Confidence (ABC) Scale and the Falls Efficacy Scale-International (FES-I). MS-related disability was assessed with the Expanded Disability Status Scale (EDSS). Relationships between the magnitude (root mean square, range, and area) as well as velocity of center of pressure sway (calculated from the center of pressure signal from the SOT), composite SOT, ABC Scale, FES-I, and EDSS scores were assessed. The magnitude and velocity of center of pressure sway was statistically significantly correlated with the ABC Scale (rho $=-0.2$ to -0.5 ), FES-I (rho $=0.3$ to 0.5 ), and EDSS (rho $=0.3$ to 0.4 ). The composite SOT was also statistically significantly correlated with the ABC Scale, FES-I, and EDSS. Objective balance measures, as reflected by posturography, were significantly related to subjective reports of imbalance and clinical measures of disability in MS. The relationships are moderate to weak, indicating that a comprehensive description of balance problems in people with MS likely requires both objective and subjective balance measures.
\end{abstract}

Key words: accidental falls, cohort studies, disability, imbalance, measurement, multiple sclerosis, physical examination, postural balance, posturography, questionnaires.

\section{INTRODUCTION}

Clinical experience and extensive research using a variety of measures indicate that balance problems are common in people with multiple sclerosis (PwMS) [1-5]. PwMS subjectively report reduced balance confidence, frequent falls, and an increased fear of falling [6-7]. Additionally, objective posturography demonstrates that multiple sclerosis (MS) is associated with balance impairments in individuals with clearly apparent disability as well as in those with minimal disability [4] or without clinically apparent disability [8]. These findings suggest that while PwMS may appear to have normal balance on clinical inspection, MS can cause changes that subtly alter postural control. Subjectively, PwMS may become aware of balance impairments when they experience changes in day-to-day performance of activities of daily living and physical activity [9].

While many objective and subjective measures have been used to examine balance in PwMS [2-3,5,10], the best measure of balance in MS has not been established, nor is it known whether it is advantageous to establish a single test. For this study, we used dynamic posturography

\footnotetext{
Abbreviations: $\mathrm{ABC}=$ Activities-specific Balance Confidence, EDSS = Expanded Disability Status Scale, FES-I = Falls Efficacy Scale-International, MRI = magnetic resonance imaging, MS = multiple sclerosis, MSWS-12 = MS Walking Scale-12, PwMS = people with MS, RMS = root mean square, SOT $=$ Sensory Organization Test.

*Address all correspondence to Michelle H. Cameron, MD, PT; Oregon Health \& Science University-Neurology, 3181 SW Sam Jackson Park Rd L226, Portland, OR 97239; 503418-1971; fax: 503-494-7289. Email: cameromi@ohsu.edu http://dx.doi.org//10.1682/JRRD.2013.02.0042
} 
to objectively quantify balance in PwMS because this is the gold standard objective measure of standing postural control and because this has been used previously to quantify balance in PwMS [3-4,11-12]. Recent posturography studies have demonstrated specific performance abnormalities on posturography in PwMS, including delayed automatic postural responses to backward translations [13] and abnormal postural sway when sensory inputs are removed [5]. For the present study, we used the composite score from the Sensory Organization Test (SOT) as generated by commercially available posturography devices and we extracted the raw center of pressure signal from each trial of the SOT and calculated root mean square (RMS), range, total sway area, and mean velocity for the resultant sway path. Analyses of these center of pressure variables have been shown to most effectively characterize different aspects of postural sway [14-15]. For example, sway area reflects the effectiveness of, or the stability achieved by, the postural control system and mean velocity reflects the amount of regulatory activity associated with this level of stability [15-16].

To quantify the subjective experience of imbalance, we used the Activities-specific Balance Confidence (ABC) Scale, which assesses fear of falling and has been shown to be reliable [2] and to distinguish between fallers and nonfallers with MS [10]. We also used the Falls Efficacy Scale-International (FES-I), which measures perceived self-efficacy at avoiding falls during essential nonhazardous activities of daily living [17].

The relationships between objective and subjective measures of balance in MS are not known. It is likely that objective and subjective measures capture different aspects of balance problems in PwMS. For example, objective assessment tools identify quantitative variables of interest related to physiological changes in vision, vestibular, and somatosensory sensory systems as well as muscular performance, which affect postural control, while self-report questionnaires likely reflect the effect of balance problems in an individual's day-to-day life. While some relationship between objective and subjective measures is expected, no single measure is likely to reflect the entirety of balance problems. This is similar to the diagnosis and assessment of disease severity and progression in MS. During the diagnosis and monitoring of MS, multiple measures, including relapse rate, various magnetic resonance imaging (MRI) measures, and disease-associated impairment and disability as captured by the Expanded Disability Status Scale (EDSS) [18], are used to assess changes in func- tion and response to treatment. Similarly, it is possible that a combination of measures to assess balance will provide the most comprehensive picture of the onset, progression, and response to treatment of balance problems in MS.

To decide whether a combination of measures is best to monitor changes in balance, it is first necessary to understand the relationship between objective and subjective balance measures. Thus, the purpose of this study was to better understand the relationships between objective measures of balance, as assessed by posturography, and subjective measures of balance, as assessed by selfrated questionnaires, in PwMS. We hypothesized that there would be relationships between these measures but that these relationships would be weak because of differences in the focus of these measures. Objective measures reflect balance performance at the specific time of testing and subjective measures reflect overall balance confidence and perceived self-efficacy at avoiding falls.

\section{MATERIALS AND METHODS}

\section{Standard Protocol Approvals, Registrations, and Patient Consents}

This was a prospective cohort study carried out at a Department of Veterans Affairs medical center and an academic medical center in the Northwest United States. The institutional review boards at both centers approved the protocol and written informed consent was obtained from all patients participating in the study. The subjects were 54 PwMS recruited from the outpatient MS specialty clinics of these centers and surrounding community neurology clinics in 2010 to 2011. Subjects were recruited for a study of mechanisms underlying imbalance and falls in MS, and the subjects included in this analysis were those who completed all measures analyzed.

\section{Participants}

Data from 54 PwMS were included in this analysis. Inclusion criteria included (1) having MS as diagnosed by McDonald criteria [19]; (2) having any subtype of MS, including relapsing remitting, primary progressive, or secondary progressive; (3) having mild-to-moderate MSrelated disability (EDSS score of $\leq 6.0$ ) [18]; (4) being willing and intellectually able to understand and sign an informed consent and adhere to protocol requirements; (5) being community dwelling; and (6) having no clinically significant MS relapses as confirmed by physician history 
within 30 days before the day of balance testing. Exclusion criteria included (1) having a self-reported musculoskeletal, neurological, or medical condition other than MS that is known to affect balance or gait and is associated with falls, such as a lower-limb joint replacement, peripheral neuropathy, vestibular disorder, alcoholism, stroke, and seizures and (2) being $>3$ mo pregnant.

\section{Objective Instrumented Quantitative Balance Assessment}

We specifically evaluated several standard measures of center of pressure sway in standing using the SOT performed with the SMART Equitest device (NeuroCom; Clackamas, Oregon) $[1,20]$. The SOT evaluates the relative contributions of visual, vestibular, and somatosensory systems during standing by disturbing the information delivered to the patient's eyes, feet, and joints. For the SOT, subjects perform three $20 \mathrm{~s}$ trials under six different conditions: (1) eyes open, (2) eyes closed, (3) eyes open with surround sway, (4) eyes open with support surface sway, (5) eyes closed with support surface sway, and (6) eyes open with surround and support surface sway
(Figure). The subject's sway under each condition reflects his or her ability to maintain postural control during standing [20]. The composite SOT score is calculated by comparing the angular difference between a subject's maximum anterior to posterior center of gravity displacements and the maximum possible sway range of $12.5^{\circ}$. The composite SOT score ranges from 0 to 100 , where 100 indicates no sway and 0 indicates a fall. Although this equilibrium score is commonly used in clinical practice, it provides no other information regarding the center of pressure sway path. Therefore, for this study, we extracted the raw center of pressure signal from each trial of the SOT and calculated RMS, range, area, and mean velocity for the resultant sway path, because these provide additional information about the stability achieved by the postural control system and the amount of regulatory activity associated with this level of stability. The median value from three trials for each condition, rather than the mean value, was used to account for any possible learning effects across trials, providing one value for each variable for each of the six conditions.

$\begin{array}{lll}\begin{array}{l}\text { Normal } \\ \text { Vision }\end{array} & \begin{array}{l}\text { Normal } \\ \text { Vision }\end{array} \\ \text { Fixed } \\ \text { Support }\end{array}$

Figure.

Sensory Organization Test. Subjects perform three 20 s trials under six different conditions: (1) eyes open, (2) eyes closed, (3) eyes open with surround sway, (4) eyes open with support surface sway, (5) eyes closed with support surface sway, (6) eyes open with surround and support surface sway. Image reprinted with permission by NeuroCom; Clackamas, Oregon. 


\section{Subjective Balance Assessment}

The ABC Scale and FES-I were completed by all subjects. The ABC Scale is a 16-item questionnaire that serves as a rating of self-perceived confidence during performance of activities of daily living. The questionnaire score ranges from 0-100 percent, with higher scores indicating higher or better balance confidence. The ABC Scale has been shown to have high test-rest reliability (Pearson correlation $=0.92$ ) and internal consistency (Cronbach $\alpha=0.96$ ) in community-dwelling older adults [21]. In PwMS, the ABC Scale also shows good test-retest reliability (Pearson correlation $=0.92$ ) [2] and concurrent validity with other objective and subjective balance measures [10,22].

The FES-I measures fear of falling, where fear of falling is defined as low perceived self-efficacy at avoiding falls during essential, nonhazardous activities of daily living [23-24]. The FES-I was used for this study because although it has not commonly been used in studies with PwMS, it has been validated in other populations and has good cross-cultural validity, unlike the $A B C$ Scale [25-26]. The FES-I also shows good test-retest reliability in community-dwelling older adults [25] and in people with cognitive impairment [23]. Subjects with higher FES-I scores have greater fear of falling [24]. Both the ABC Scale and the FES-I are subjective measures, but they have slightly different purposes. The ABC Scale focuses on assessing an individual's perception of his or her balance. The FES-I focuses on an individual's fear of falling. Thus, use of both scales provides both a balance-specific and a falls-specific self-assessment.

\section{Clinical Impairment and Disability Assessment}

Clinical assessment of overall MS-associated impairments and disability was performed using the EDSS [18]. The EDSS quantifies impairments and disability in MS in eight functional systems: pyramidal, cerebellar, brainstem, sensory, bowel and bladder, visual, cerebral, and other. Based on the functional system score, the person is classified into an EDSS step or half step between 0 and 10 , with 0 reflecting a normal neurological examination, 10.0 reflecting death due to MS, and 6.0 reflecting intermittent or unilateral constant assistance (cane, crutch, or brace) required to walk $100 \mathrm{~m}$. The EDSS is the scale most commonly used to rate disability in MS. In the present study, the EDSS assessment was performed by a qualified neurologist or nurse practitioner.

\section{Data Analysis}

Means (or medians where appropriate), ranges, and standard deviations for demographic and balance questionnaire scores were calculated by standard methods and are shown in Table 1. To assess the relationship between sway variables measured by the SOT and scores on the ABC Scale, FES-I, and EDSS, Spearman correlations were computed after a Shapiro-Wilks test revealed the data were not normally distributed. A Bonferroni correction for multiple comparisons was applied so the alpha level was set at $0.008(0.05 / 6$, for the 6 conditions). All statistical procedures were performed using SPSS (SPSS version 20, IBM Corp; Armonk, New York).

\section{RESULTS}

The subjects had an average age of $39.5 \mathrm{yr}, 70$ percent were female, and their median EDSS score was 3.0 (Table 1). The mean ABC Scale and FES-I scores were 79.3 and 24.8, respectively (Table 1).

ABC Scale total scores significantly correlated with median values for RMS in conditions 1,2 , and 3; with range in conditions 1 and 3; with velocity in conditions 1-5; and with sway area in all SOT conditions (Table 2). FES-I total scores significantly correlated with median values for RMS in conditions 1 and 3; with range in conditions 1,3 , and 4; and with velocity and sway area in all SOT conditions (Table 3). EDSS scores significantly correlated with median values for RMS in conditions 1 , 5 , and 6; with range in conditions 1 and 6; with velocity in conditions 1 and 4 ; and with sway area in conditions 1 ,

Table 1.

Subject demographics.

\begin{tabular}{lc}
\hline \multicolumn{1}{c}{ Variable } & Mean \pm SD \\
\hline Age (yr) & $39.5 \pm 8.4$ \\
Sex (female/male) & $38 / 16$ \\
EDSS (median) & 3.0 \\
EDSS (range) & $0.0-6.0$ \\
Time Since Symptom Onset (yr) & $11.7 \pm 8.2$ \\
Time Since Diagnosis (yr) & $7.6 \pm 5.9$ \\
MS Subtype (RRMS/SPMS) & $51 / 3$ \\
ABC Scale (score) & $79.3 \pm 21.0$ \\
FES-I (score) & $24.8 \pm 8.5$ \\
ABC = Activities-specific Balance Confidence, EDSS = Expanded Disability \\
Status Scale, FES-I = Falls Efficacy Scale-International, MS = multiple sclerosis, \\
RRMS = relapsing-remitting MS, SD = standard deviation, SPMS = secondary- \\
progressive MS.
\end{tabular}


Table 2.

Correlations between sway measures and Activities-specific Balance Confidence Scale.

\begin{tabular}{|c|c|c|c|c|c|c|c|c|}
\hline \multirow{2}{*}{ Condition ${ }^{*}$} & \multicolumn{2}{|c|}{ RMS } & \multicolumn{2}{|c|}{ Range } & \multicolumn{2}{|c|}{ Velocity } & \multicolumn{2}{|c|}{ Area } \\
\hline & rho & $p$-Value ${ }^{\dagger}$ & rho & $p$-Value ${ }^{\dagger}$ & rho & $p$-Value ${ }^{\dagger}$ & rho & $p$-Value ${ }^{\dagger}$ \\
\hline 1 & -0.46 & $<0.001$ & -0.44 & 0.001 & -0.48 & $<0.001$ & -0.49 & $<0.001$ \\
\hline 2 & -0.34 & $<0.001$ & -0.33 & 0.01 & -0.37 & 0.007 & -0.40 & 0.003 \\
\hline 3 & -0.45 & 0.001 & -0.49 & $<0.001$ & -0.38 & 0.004 & -0.47 & $<0.001$ \\
\hline 4 & -0.25 & 0.06 & -0.28 & 0.04 & -0.42 & 0.002 & -0.40 & 0.003 \\
\hline 5 & -0.28 & 0.04 & -0.27 & 0.04 & -0.38 & 0.004 & -0.46 & 0.001 \\
\hline 6 & -0.23 & 0.10 & -0.25 & 0.07 & -0.35 & 0.01 & -0.38 & 0.004 \\
\hline
\end{tabular}

${ }^{*} 1$ = eyes open, 2 = eyes closed, 3 = eyes open with surround sway, 4 = eyes open with support surface sway, 5 = eyes closed with support surface sway, 6 = eyes open with surround and support surface sway.

${ }^{\dagger}$ Bold indicates significance at $p \leq 0.008$.

$\mathrm{RMS}=$ root mean square.

Table 3.

Correlations between sway measures and Falls Efficacy Scale-International.

\begin{tabular}{|c|c|c|c|c|c|c|c|c|}
\hline \multirow{2}{*}{ Condition ${ }^{*}$} & \multicolumn{2}{|c|}{ RMS } & \multicolumn{2}{|c|}{ Range } & \multicolumn{2}{|c|}{ Velocity } & \multicolumn{2}{|c|}{ Area } \\
\hline & rho & $p$-Value ${ }^{\dagger}$ & rho & $p$-Value ${ }^{\dagger}$ & rho & $p$-Value ${ }^{\dagger}$ & rho & $p$-Value ${ }^{\dagger}$ \\
\hline$\overline{1}$ & 0.42 & 0.002 & 0.42 & 0.001 & 0.47 & $<0.001$ & 0.48 & $<0.001$ \\
\hline 2 & 0.35 & 0.01 & 0.35 & 0.009 & 0.38 & 0.004 & 0.42 & 0.002 \\
\hline 3 & 0.50 & $<0.001$ & 0.52 & $<0.001$ & 0.38 & 0.004 & 0.49 & $<0.001$ \\
\hline 4 & 0.32 & 0.02 & 0.37 & 0.007 & 0.47 & $<0.001$ & 0.47 & $<0.001$ \\
\hline 5 & 0.34 & 0.01 & 0.33 & 0.01 & 0.41 & 0.002 & 0.44 & $<0.001$ \\
\hline 6 & 0.28 & 0.04 & 0.30 & 0.03 & 0.37 & 0.007 & 0.41 & 0.002 \\
\hline
\end{tabular}

* 1 = eyes open, 2 = eyes closed, 3 = eyes open with surround sway, 4 = eyes open with support surface sway, 5 = eyes closed with support surface sway, 6 = eyes open with surround and support surface sway.

†Bold indicates significance at $p \leq 0.008$.

$\mathrm{RMS}=$ root mean square

4, 5 , and 6 of the SOT (Table 4). SOT composite scores were significantly correlated with scores on ABC Scale (rho $=0.39, p=0.004)$, FES-I (rho $=-0.43, p=0.001$ ), and EDSS ( $\mathrm{rho}=-0.36, p=0.008)$.

\section{DISCUSSION}

Results of this study indicate that objective posturography measures of balance are related to subjective selfreported measures of balance problems and clinical measures of impairment and disability in PwMS. The relationships, although statistically significant, are weak to moderate (rho $=0.33$ to 0.55 ).

Our finding of relationships between objective and subjective assessments of balance control in PwMS is consistent with Motl and Snook's [27] recent study examining the relationship between objective and subjective assessments of walking in PwMS. The authors reported a strong correlation $(r=-0.64)$ between walking performance measured objectively with an accelerometer, the gold standard objective measure of ambulation activity in individuals with neurological diseases [28], and self-reports of walking performance in PwMS (as captured by the MS Walking Scale-12 [MSWS-12] [29]). PwMS who walked more, based on accumulation of daily activity counts by accelerometry, reported less effect of the disease on walking [27]. Similarly, the present study found that PwMS who had better balance, based on center of pressure variables captured by posturography, reported greater balance confidence and less fear of falling. The previously reported relationships between objective and subjective measures of walking were stronger than those found between objective and subjective measures of balance. This difference may be 
Table 4.

Correlations between sway measures and Expanded Disability Status Scale.

\begin{tabular}{|c|c|c|c|c|c|c|c|c|}
\hline \multirow{2}{*}{ Condition $^{*}$} & \multicolumn{2}{|c|}{ RMS } & \multicolumn{2}{|c|}{ Range } & \multicolumn{2}{|c|}{ Velocity } & \multicolumn{2}{|c|}{ Area } \\
\hline & rho & $p$-Value ${ }^{\dagger}$ & rho & $p$-Value ${ }^{\dagger}$ & rho & $p$-Value ${ }^{\dagger}$ & rho & $p$-Value ${ }^{\dagger}$ \\
\hline$\overline{1}$ & 0.40 & 0.003 & 0.43 & 0.001 & 0.42 & 0.002 & 0.42 & 0.001 \\
\hline 2 & 0.28 & 0.04 & 0.26 & 0.06 & 0.23 & 0.10 & 0.33 & 0.01 \\
\hline 3 & 0.35 & 0.009 & 0.35 & 0.009 & 0.20 & 0.16 & 0.34 & 0.01 \\
\hline 4 & 0.26 & 0.05 & 0.29 & 0.03 & 0.40 & 0.008 & 0.40 & 0.003 \\
\hline 5 & 0.37 & 0.006 & 0.35 & 0.01 & 0.33 & 0.01 & 0.44 & 0.001 \\
\hline 6 & 0.37 & 0.006 & 0.37 & 0.006 & 0.33 & 0.02 & 0.44 & 0.001 \\
\hline
\end{tabular}

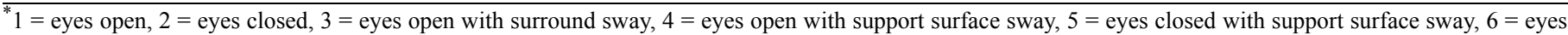
open with surround and support surface sway.

${ }^{\dagger}$ Bold indicates significance at $p \leq 0.008$.

$\underline{\mathrm{RMS}}=\underline{\mathrm{root} \text { mean square. }}$

because the subjective walking scale used, the MSWS-12, was specifically designed, validated, and optimized in PwMS, while the subjective balance scales used in the present study were originally designed for and validated in populations of older adults. It is also possible that the relationships between subjective and objective balance measures were weak because posturography captures balance performance, while the ABC Scale and FES-I assess perceived balance confidence and perceived self-efficacy at avoiding falls. Thus, the constructs differ between these measures.

The center of pressure variables used in this study reflect both the balance control processes necessary to maintain stance and movement of the entire body's center of mass [14]. Previous studies have shown that even when standing with eyes open, PwMS exhibit a larger center of pressure sway area [4,30], greater sway velocity $[3,30]$, and greater RMS of sway than nondisabled controls [31-32]. The direction of correlations found in the present study suggest that the PwMS who report poor balance also have higher sway RMS, range, velocity, and area. This finding is similar to the finding in older adults that subjective measures of balance, including the $A B C$ Scale, correlate strongly with objective measures of balance control [33]. However, the relationships between sway variables and self-report scales in this study were only weak to moderate. This is likely because factors other than sway characteristics, including fall prevention strategies, the environment, and overall confidence, modulate balance confidence and falling. In addition, correlations are likely higher in older adults because both the subjective balance scales were designed for and validated in that population $[21,26]$.

The ABC Scale represents a person's rating of their own confidence performing activities of daily living, including tasks involving walking, standing, and upperlimb movements. Postural sway may strongly affect performance of some of these tasks, such as standing on an unfamiliar surface or standing on one's toes and reaching for something but may have less effect on other more gait-related tasks, such as walking outside to the car or walking up and down a ramp, all activities rated by the ABC Scale. Similar to the ABC Scale, the FES-I identifies a person's self-perceived fear of falling while performing activities of daily living. While excessive sway during stance could be noticed by PwMS, it is also likely that a person's previous experience with falls or physical impairments would affect his or her self-perceived risk and fear of falling.

The weak relationship between the EDSS scores and sway variables is likely because although some aspects of disability that affect the EDSS score may also affect balance, many of the assessed functions, for example, speech or bladder function, would not be likely to affect balance performance. Balance is only directly assessed within the EDSS by the Romberg test [34] and observation for ataxia during ambulation. Neither of these assessment techniques, however, include a direct measure of postural sway. Thus, the EDSS score provides an overall measure of MS-associated impairment and disability that may or may not be associated with postural sway abnormalities.

The findings of this study have a number of clinical implications. Most importantly, to fully understand the 
balance problems of PwMS and to develop the ideal intervention plan, both objective and subjective balance measures should be used. Objective measures may be posturography or, when posturography equipment is not available, other clinical balance measures validated in PwMS, such as the Four Square Step Test [35], Timed "Up and Go" test [36], or the Berg Balance Scale [37], could be considered. Ideal subjective measures are generally well-designed and validated questionnaires. In addition, the clinician should realize that although those with greater impairment and disability, as reflected by higher EDDS scores, will likely have poorer balance, even those with minimal impairment and low EDSS scores may have significant imbalance.

\section{CONCLUSIONS}

The relationship between sway variables and these commonly used self-report and clinical measures provides a clear example of the imperfect relationship between available objective information regarding balance and the subjective data provided by questionnaires or general neurological clinical examination. Just as multiple measures, including MRI, relapse rate, and disability progression, are needed to encompass the severity of MS and the effectiveness of MS disease-modifying interventions, multiple measures, including self-report questionnaires, clinical examination, and posturography, are also required to effectively provide the most encompassing evaluation of balance impairment in PwMS. Both objective and subjective measures should be used to assess the presence and severity of imbalance as well as the response to interventions in clinical and experimental settings.

\section{ACKNOWLEDGMENTS}

\author{
Author Contributions: \\ Study concept and design: M. H. Cameron, J. Huisinga. \\ Acquisition of data: M. H. Cameron. \\ Analysis and interpretation of data: M. H. Cameron, J. Huisinga. \\ Statistical analysis: J. Huisinga. \\ Drafting of manuscript: M. H. Cameron, J. Huisinga. \\ Critical revision of manuscript for important intellectual content: \\ M. H. Cameron, J. Huisinga. \\ Obtained funding: M. H. Cameron. \\ Study supervision: M. H. Cameron.
}

Financial Disclosures: The authors have declared that no competing interests exist.
Funding/Support: This material was based on work supported by the Department of Veterans Affairs Rehabilitation Research and Development Service (grant VA 05-2509).

Institutional Review: The institutional review boards at both centers approved the protocol and written informed consent was obtained from all patients participating in the study.

Participant Follow-Up: The authors plan to inform participants of the publication of this and other articles resulting from this study.

\section{REFERENCES}

1. Jackson RT, Epstein CM, De l'Aune WR. Abnormalities in posturography and estimations of visual vertical and horizontal in multiple sclerosis. Am J Otol. 1995;16(1):88-93. [PMID:8579184]

2. Cattaneo D, Jonsdottir J, Repetti S. Reliability of four scales on balance disorders in persons with multiple sclerosis. Disabil Rehabil. 2007;29(24):1920-25. [PMID:17852286] http://dx.doi.org/10.1080/09638280701191859

3. Sosnoff JJ, Socie MJ, Boes MK, Sandroff BM, Pula JH, Suh Y, Weikert M, Balantrapu S, Morrison S, Motl RW. Mobility, balance and falls in persons with multiple sclerosis. PLoS ONE. 2011;6(11):e28021. [PMID:22132196] http://dx.doi.org/10.1371/journal.pone.0028021

4. Van Emmerik RE, Remelius JG, Johnson MB, Chung LH, Kent-Braun JA. Postural control in women with multiple sclerosis: Effects of task, vision and symptomatic fatigue. Gait Posture. 2010;32(4):608-14. [PMID:20943393] http://dx.doi.org/10.1016/j.gaitpost.2010.09.002

5. Cattaneo D, Jonsdottir J. Sensory impairments in quiet standing in subjects with multiple sclerosis. Mult Scler. 2009;15(1):59-67.

6. Finlayson ML, Peterson EW, Cho CC. Risk factors for falling among people aged 45 to 90 years with multiple sclerosis. Arch Phys Med Rehabil. 2006;87(9):1274-79, quiz 1287. [PMID:16935067] http://dx.doi.org/10.1016/j.apmr.2006.06.002

7. Peterson EW, Cho CC, von Koch L, Finlayson ML. Injurious falls among middle aged and older adults with multiple sclerosis. Arch Phys Med Rehabil. 2008;89(6):1031-37. [PMID: 18503796] http://dx.doi.org/10.1016/j.apmr.2007.10.043

8. Martin CL, Phillips BA, Kilpatrick TJ, Butzkueven H, Tubridy N, McDonald E, Galea MP. Gait and balance impairment in early multiple sclerosis in the absence of clinical disability. Mult Scler. 2006;12(5):620-28.

9. Nilsagård Y, Denison E, Gunnarsson LG, Boström K. Factors perceived as being related to accidental falls by persons with multiple sclerosis. Disabil Rehabil. 2009;31(16):1301-10. [PMID:19479575] http://dx.doi.org/10.1080/09638280802532639 
10. Cattaneo D, Regola A, Meotti M. Validity of six balance disorders scales in persons with multiple sclerosis. Disabil Rehabil. 2006;28(12):789-95. [PMID:16754576] http://dx.doi.org/10.1080/09638280500404289

11. Chung LH, Remelius JG, Van Emmerik RE, Kent-Braun JA. Leg power asymmetry and postural control in women with multiple sclerosis. Med Sci Sports Exerc. 2008;40(10): 1717-24. [PMID:18799980] http://dx.doi.org/10.1249/MSS.0b013e31817e32a3

12. Huisinga JM, Filipi ML, Stergiou N. Supervised resistance training results in changes in postural control in patients with multiple sclerosis. Motor Control. 2012;16(1):50-63. [PMID:22402220]

13. Cameron MH, Horak FB, Herndon RR, Bourdette D. Imbalance in multiple sclerosis: A result of slowed spinal somatosensory conduction. Somatosens Mot Res. 2008; 25(2):113-22. [PMID:18570015] http://dx.doi.org/10.1080/08990220802131127

14. Rocchi L, Chiari L, Cappello A. Feature selection of stabilometric parameters based on principal component analysis. Med Biol Eng Comput. 2004;42(1):71-79.

[PMID:14977225]

http://dx.doi.org/10.1007/BF02351013

15. Mancini M, Horak FB, Zampieri C, Carlson-Kuhta P, Nutt JG, Chiari L. Trunk accelerometry reveals postural instability in untreated Parkinson's disease. Parkinsonism Relat Disord. 2011;17(7):557-62. [PMID:21641263] http://dx.doi.org/10.1016/j.parkreldis.2011.05.010

16. Maki BE, Holliday PJ, Topper AK. Fear of falling and postural performance in the elderly. J Gerontol. 1991;46(4): M123-31. [PMID:2071833] http://dx.doi.org/10.1093/geronj/46.4.M123

17. Yardley L, Beyer N, Hauer K, Kempen G, Piot-Ziegler C, Todd C. Development and initial validation of the Falls Efficacy Scale-International (FES-I). Age Ageing. 2005; 34(6):614-19. [PMID:16267188] http://dx.doi.org/10.1093/ageing/afi196

18. Kurtzke JF. Rating neurologic impairment in multiple sclerosis: An expanded disability status scale (EDSS). Neurology. 1983;33(11):1444-52. [PMID:6685237] http://dx.doi.org/10.1212/WNL.33.11.1444

19. Polman CH, Reingold SC, Edan G, Filippi M, Hartung HP, Kappos L, Lublin FD, Metz LM, McFarland HF, O’Connor PW, Sandberg-Wollheim M, Thompson AJ, Weinshenker BG, Wolinsky JS. Diagnostic criteria for multiple sclerosis: 2005 revisions to the "McDonald Criteria". Ann Neurol. 2005;58(6):840-46. [PMID:16283615] http://dx.doi.org/10.1002/ana.20703

20. Kasser SL, Jacobs JV, Foley JT, Cardinal BJ, Maddalozzo GF. A prospective evaluation of balance, gait, and strength to predict falling in women with multiple sclerosis. Arch Phys Med Rehabil. 2011;92(11):1840-46.

\section{[PMID:21840497]}

http://dx.doi.org/10.1016/j.apmr.2011.06.004

21. Powell LE, Myers AM. The Activities-specific Balance Confidence (ABC) Scale. J Gerontol A Biol Sci Med Sci. 1995;50A(1):M28-34. [PMID:7814786]

http://dx.doi.org/10.1093/gerona/50A.1.M28

22. Nilsagård Y, Carling A, Forsberg A. Activities-specific balance confidence in people with multiple sclerosis. Mult Scler Int. 2012;2012:613925. [PMID:22919491]

http://dx.doi.org/10.1155/2012/613925

23. Hauer K, Yardley L, Beyer N, Kempen G, Dias N, Campbell M, Becker C, Todd C. Validation of the Falls Efficacy Scale and Falls Efficacy Scale International in geriatric patients with and without cognitive impairment: Results of self-report and interview-based questionnaires. Gerontology. 2010;56(2):190-99. [PMID:19729878] http://dx.doi.org/10.1159/000236027

24. Tinetti ME, Richman D, Powell L. Falls efficacy as a measure of fear of falling. J Gerontol. 1990;45(6):239-43.

[PMID:2229948]

http://dx.doi.org/10.1093/geronj/45.6.P239

25. Helbostad JL, Taraldsen K, Granbo R, Yardley L, Todd CJ, Sletvold O. Validation of the Falls Efficacy ScaleInternational in fall-prone older persons. Age Ageing. 2010;39(2):259. [PMID:20031925] http://dx.doi.org/10.1093/ageing/afp224

26. Delbaere K, Close JC, Mikolaizak AS, Sachdev PS, Brodaty H, Lord SR. The Falls Efficacy Scale International (FES-I). A comprehensive longitudinal validation study. Age Ageing. 2010;39(2):210-16. [PMID:20061508] http://dx.doi.org/10.1093/ageing/afp225

27. Motl RW, Snook EM. Confirmation and extension of the validity of the Multiple Sclerosis Walking Scale-12 (MSWS-12). J Neurol Sci. 2008;268(1-2):69-73.

[PMID:18061618] http://dx.doi.org/10.1016/j.jns.2007.11.003

28. Pearson OR, Busse ME, van Deursen RW, Wiles CM. Quantification of walking mobility in neurological disorders. QJM. 2004;97(8):463-75. [PMID:15256604] http://dx.doi.org/10.1093/qjmed/hch084

29. Hobart JC, Riazi A, Lamping DL, Fitzpatrick R, Thompson AJ. Measuring the impact of MS on walking ability: the 12-Item MS Walking Scale (MSWS-12). Neurology. 2003; 60(1):31-36. [PMID:12525714] http://dx.doi.org/10.1212/WNL.60.1.31

30. Sosnoff JJ, Shin S, Motl RW. Multiple sclerosis and postural control: The role of spasticity. Arch Phys Med Rehabil. 2010;91(1):93-99. [PMID:20103402] http://dx.doi.org/10.1016/j.apmr.2009.09.013

31. Huisinga JM, Filipi M, Stergiou N. Supervised resistance training results in changes in postural control in patients 
with multiple sclerosis. Mot Contr. 2012;16(1):50-63. [PMID:22402220]

32. Huisinga JM, Yentes JM, Filipi ML, Stergiou N. Postural control strategy during standing is altered in patients with multiple sclerosis. Neurosci Lett. 2012;524(2):124-28. [PMID:22824302] http://dx.doi.org/10.1016/j.neulet.2012.07.020

33. Portegijs E, Edgren J, Salpakoski A, Kallinen M, Rantanen T, Alen M, Kiviranta I, Sihvonen S, Sipilä S. Balance confidence was associated with mobility and balance performance in older people with fall-related hip fracture: A crosssectional study. Arch Phys Med Rehabil. 2012;93(12): 2340-46. [PMID:22698990] http://dx.doi.org/10.1016/j.apmr.2012.05.022

34. Khasnis A, Gokula RM. Romberg's test. J Postgrad Med. 2003;49(2):169-72. [PMID:12867698]

35. Dite W, Temple VA. A clinical test of stepping and change of direction to identify multiple falling older adults. Arch Phys Med Rehabil. 2002;83(11):1566-71. [PMID:12422327] http://dx.doi.org/10.1053/apmr.2002.35469
36. Podsiadlo D, Richardson S. The timed "Up \& Go": A test of basic functional mobility for frail elderly persons. J Am Geriatr Soc. 1991;39(2):142-48. [PMID:1991946]

37. Berg KO, Wood-Dauphinee SL, Williams JI, Maki B. Measuring balance in the elderly: Validation of an instrument. Can J Public Health. 1992;83(Suppl 2):S7-11.

[PMID:1468055]

Submitted for publication February 12, 2013. Accepted in revised form June 13, 2013.

This article and any supplementary material should be cited as follows:

Cameron MH, Huisinga J. Objective and subjective measures reflect different aspects of balance in multiple sclerosis. J Rehabil Res Dev. 2013;50(10):1401-10. http://dx.doi.org/10.1682/JRRD.2013.02.0042

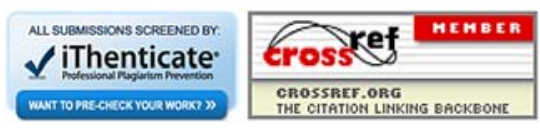


